\title{
On the fracture toughness of advanced materials $^{* *}$
}

\author{
By Maximilien E. Launey and Robert O. Ritchie*
}

\begin{abstract}
Few engineering materials are limited by their strength; rather they are limited by their resistance to fracture or fracture toughness. It is not by accident that most critical structures, such as bridges, ships, nuclear pressure vessels and so forth, are manufactured from materials that are comparatively low in strength but high in toughness. Indeed, in many classes of materials, strength and toughness are almost mutually exclusive. In the first instance, such resistance to fracture is a function of bonding and crystal structure (or lack thereof), but can be developed through the design of appropriate nano/microstructures. However, the creation of tough microstructures in structural materials, i.e., metals, polymers, ceramics and their composites, is invariably a compromise between resistance to intrinsic damage mechanisms ahead of the tip of a crack (intrinsic toughening) and the formation of crack-tip shielding mechanisms which principally act behind the tip to reduce the effective "crack-driving force" (extrinsic toughening). Intrinsic toughening is essentially an inherent property of a specific microstructure; it is the dominant form of toughening in ductile (e.g., metallic) materials. However, for most brittle (e.g., ceramic) solids, and this includes many biological materials, it is largely ineffective and toughening conversely must be developed extrinsically, by such shielding mechanisms as crack bridging. From a fracture mechanics perspective, this results in toughening in the form of rising resistance-curve behavior where the fracture resistance actually increases with crack extension. The implication of this is that in many biological and high-strength advanced materials, toughness is developed primarily during crack growth and not for crack initiation. This is an important realization yet is still rarely reflected in the way that toughness is measured, which is invariably involves the use of single-value (crack-initiation) parameters such as the fracture toughness $K_{\text {Ic. }}$
\end{abstract}

\footnotetext{
[*] Prof. Robert O. Ritchie

Department of Materials Science and Engineering, University of California Berkeley, and Materials

Sciences Division, Lawrence Berkeley National Laboratory, Berkeley, California, 94720 (USA)

E-mail: RORitchie@lbl.gov

Dr. Maximilien E. Launey

Materials Sciences Division, Lawrence Berkeley National Laboratory, Berkeley, California, 94720 (USA)

E-mail: MELauney@lbl.gov

[**] The authors acknowledge financial support from the Director, Office of Science, Office of Basic Energy Sciences, Division of Materials Sciences and Engineering, of the U.S. Department of Energy under Contract No. DE-AC02-05CH11231.
} 


\section{Introduction: strength vs. toughness}

A fundamental tenet of materials science is that the mechanical properties of materials are a function of their structure, specifically their short- and long-range atomic structure and at higher dimensions their nano/microstructure. In this regard, there has been much activity in recent years focused on developing materials with much higher strength, for example through the use of finer-scale structures and/or reinforcements (nano-materials, nano-structures, nanocomposites, etc). The motivation for this is to be able to use smaller section sizes, with a consequent reduction in weight or fuel consumption or whatever the application happens to be. However, as it is often the case, there is a "gulf" between scientific deliberations and engineering practice - few (bulk) materials that we currently use in critical structural applications are specifically chosen for their strength; more often than not, a much more important concern is their toughness, i.e., their resistance to fracture. Unfortunately, although these properties may seem to many to be similar, changes in material structure often affect the strength and toughness in very different ways.

From the perspective of atomic structure and bonding, it has long been known that high strength can be associated with strong directional bonding, high Peierls-Nabarro forces and (in crystalline solids) limited dislocation mobility, yet this invariably is a recipe for brittle behavior and poor toughness. Similarly, at larger size-scales, microstructures which restrict plasticity (or more generally inelasticity) will display high strength properties, but this again can lead to lower toughness by minimizing the local relief of high stresses, e.g., by crack-tip blunting. Indeed, although there are exceptions, toughness is usually inversely proportional to strength, such that the design of strong and tough materials is inevitably a compromise.

Since structural materials are often used in applications where catastrophic fracture is not an option, such as for nuclear containment vessels, aircraft jet engines, gas pipelines, even critical medical implants like cardiovascular stents and heart valve prostheses, it can be usefully argued that the property of toughness is far more important than strength. Accordingly, recognizing the necessary trade-offs in microstructures, one would expect that research on modern (bulk) structural materials would be increasingly tailored to achieving an optimum combination of these two properties. Unfortunately, this is rarely the case, and much physics- and materials-based research is still too focused on 
the quest for higher strength ${ }^{[*]}$ without any corresponding regard for toughness. A notable exception here is in the ceramics community, where the extreme brittleness of ceramic materials has necessitated a particular emphasis on the issue of fracture resistance and toughness.

In ductile materials such as metals and polymers, strength is a measure of the resistance to permanent (plastic) deformation. It is defined, invariably in uniaxial tension, compression or bending, either at first yield (yield strength) or at maximum load (ultimate strength). The general rule with metals and alloys is that the toughness is inversely proportional to the strength. A notable exception is certain aluminum alloys, e.g., Al-Li alloys, which are significantly tougher at liquid helium temperatures (where they naturally display higher strength). This results from their tendency at low temperatures to form delamination cracks in the through-thickness (short-transverse) direction; the toughness is then elevated by "delamination toughening" in the longitudinal (crack-divider) orientation, where the material effectively splits in several higher toughness, plane-stress sections, and by crack arrest at the delamination cracks in the transverse (crackarrester) orientation. ${ }^{\left.[2]]^{* *}\right]}$

In brittle materials such as ceramics, where at low homologous temperatures macroscopic plastic deformation is essentially absent, the strength measured in uniaxial tension or bending is governed by when the sample fractures. Strength, however, does not necessarily provide a sound assessment of toughness as it cannot define the relative contribution of flaws and defects from which fracture invariably ensues. For this reason, strength and toughness can also be inversely related in ceramics. By way of example, refining the grain size can limit the size of pre-existing microcracks, which is beneficial for strength, yet for fracture mechanics based toughness measurements, where the test samples already contain a worst-case crack, the smaller grain size provides less resistance to crack extension, generally by reducing the potency of any grain bridging, which lowers the toughness.

${ }^{[*}$ A good example here is the excitement generated by the discovery of carbon nanotubes which have exceptionally high strengths better than $E / 10$ ( $E$ is Young's modulus).[1] However, it is still uncertain whether such nanotubes can be harnessed into bulk structural materials which can utilize their high strength (and stiffness) without compromising toughness.

[**] Because of this toughening behavior, Al-Li alloys are used for the main fuel tank of the Space Shuttle. A similar approach of "delamination toughening" has recently been used as a means to toughen steels. ${ }^{[3]}$ 
Flaws in materials are either microstructural in origin, e.g., microcracks or microvoids formed at inclusions, brittle second-phase particles and grainboundary films, or introduced during handing, synthesis and processing, such as porosity, shrinkage cavities, quench cracks, grinding and stamping marks (such as gouges, burns, tears, scratches, and cracks), seams and weld-related cracks. Their relevance and statistical consequence was first demonstrated several centuries ago by Leonardo da Vinci. ${ }^{[4]}$ He measured the strength of brittle iron wires and found that the fracture strength was not a constant like the yield strength but rather varied inversely with wire length, implying that flaws in the material controlled the strength; a longer wire resulted in a larger sampling volume and thus provided a higher probability of finding a significant flaw. This dependence of strength on the pre-existing flaw distributions has several important implications for brittle materials. In particular, large specimens tend to have lower strengths than smaller ones, and specimens tested in tension tend to have lower strengths than identically-sized specimens tested in bending because the volume (and surface area) of material subjected to peak stresses is much larger; in both cases, the lower strength is associated with a higher probability of finding a larger flaw.

Corresponding quantitative descriptions of the toughness fall in the realm of fracture mechanics, which in many ways began with the work of Griffith on fracture in glass in the 1920's, but was formally developed by Irwin and others from the late 1940s onwards. As opposed to the strength of materials approach, fracture mechanics considers the flaw size as an additional structural variable, and the fracture toughness replaces strength as the relevant material property. With certain older measures of toughness, such as the work to fracture or Charpy V-notch energy, which are determined by breaking an unnotched or rounded notched sample, the toughness and strength in a brittle material are essentially evaluating the same property (although the units may be different). The conclusion here is that for all classes of materials, the fracture resistance does not simply depend upon the maximum stress or strain to cause fracture but also on the ubiquitous presence of crack-like defects and their size. Since the preexisting defect distribution is rarely known in strength tests, the essence of the fracture mechanics description of toughness is to first pre-crack the test sample to create a known (nominally atomically-sharp) worst-case crack, and then to 
determine the stress intensity or energy required, i.e., the fracture toughness,${ }^{[*}$ to fracture the material in the presence of this worst-case flaw.

Here we describe the some of the differing toughening mechanisms prevalent both in engineering (metals, ceramics, polymers and composites) and biological (bone, nacre, wood) materials. We further describe how by using Nature's inspiration of hierarchical structural architectures, advanced composite (hybrid) materials can be developed (in bulk form) with strength and toughness properties far superior to those of their individual constituents.

\section{Toughening mechanisms}

\subsection{Intrinsic $v s$. extrinsic toughening ${ }^{[6]}$}

Traditionally, toughness has been thought of as the ability of a material to dissipate deformation energy without propagation of a crack. In fracture mechanics terms, however, the initiation and subsequent extension of a crack can be considered, specifically in terms of the "crack-driving force" (e.g., $K, \boldsymbol{g}$ or $J$ ) opposed by the resistance of the microstructure. Toughness can be enhanced by increasing the microstructural resistance, such as by changing the nature, distribution and/or interface properties of second-phase particles to suppress damage in the form of microcracking or microvoid formation ahead of the crack tip; this is termed intrinsic toughening and is the principal means by which ductile materials, e.g., metallic materials, derive their toughness. However, this approach is largely ineffective with brittle materials such as ceramics, ${ }^{[7]}$ which invariably must rely on extrinsic toughening. Extrinsic toughening involves microstructural mechanisms that act primarily behind the crack tip to effectively reduce the crack-driving force actually experienced at the crack tip; this is termed crack-tip shielding and can occur by such mechanisms as crack bridging and in

\footnotetext{
${ }^{\left[{ }^{*}\right]}$ Under linear elastic deformation conditions, the fracture toughness, $K_{c}$, is the critical value of the stress intensity $K$ for unstable fracture at a pre-existing crack, i.e., when $K=Y \sigma_{\text {app }}(\pi a)^{1 / 2}=K_{c}$, where $\sigma_{\mathrm{app}}$ is the applied stress (equal to the fracture stress, $\sigma_{\mathrm{F}}$, at criticality), $a$ is the crack length (equal to the critical crack size, $a_{c}$, at criticality), and $Y$ is a function (of order unity) of crack size and geometry. Alternatively, the toughness can be expressed as a critical value of the strain energy release rate, $\mathscr{q}_{c}$, defined as the change in potential energy per unit increase in crack area, i.e., when $\mathcal{G}_{c}=K_{\mathrm{c}}^{2} / E^{\prime}$, where $E^{\prime}$ is the appropriate elastic modulus. Under nonlinear elastic conditions, where the degree of plasticity is more extensive, an analogous nonlinear elastic fracture mechanics approach may be used based on the J-integral, which is the nonlinear elastic energy release rate and hence equivalent to $\mathcal{G}$ under linear elastic conditions. ${ }^{[5]}$
} 
situ phase transformations. Indeed, fracture is the result of a mutual competition of intrinsic (damage) mechanisms ahead of the crack tip that promote cracking and extrinsic (shielding) mechanisms mainly behind the tip trying to impede it (Fig. 1). ${ }^{[6]}$

Intrinsic toughening mechanisms are an inherent property of the material, and thus are active irrespective of crack size and geometry; they affect primarily the initiation but also the growth of a crack. Extrinsic mechanisms, conversely, act in the crack wake and are thus dependent on crack size (and to some degree specimen geometry). Consequently, they result in crack-size dependent fracture behavior, a principal manifestation of which is resistance-curve (R-curve) toughness behavior where the crack-driving force to sustain cracking increases with crack extension (Fig. 2). Extrinsic toughening mechanisms affect only the crack growth toughness; they have little effect on crack initiation.

\subsection{Toughening in structural engineering materials}

Metallic materials: As noted above, metals develop their toughness primarily from intrinsic mechanisms, with crack-tip plasticity as the dominating factor. With fracture, plastic deformation acts to reduce the stress intensification at the crack tip by inducing crack-tip blunting through the emission of dislocations (ductile behavior), as opposed to local decohesion by simply breaking an atomic bond there (brittle behavior). The subsequent interaction of dislocations with microstructural inhomogeneities in metals then provides the mechanisms of damage, in the form of pile-ups at grain boundaries, or at second-phase particles causing them to crack or decohere from the matrix. Depending on such factors as the flow stress (which is a function of temperature and strain rate) and level of constraint (which depends on stress-state and specimen geometry), the resulting fracture be associated with only limited plastic deformation and be (nominally) brittle, i.e., transgranular cleavage or intergranular fracture, which are locally stress-controlled and invariably lead to low toughness, or involve more extensive plastic deformation which leads to failure by ductile, i.e., microvoid coalescence, fracture, which is locally strain-controlled and generally results in much higher toughness.

Analogous to the glass transition temperature in polymers, mechanically brittle fracture in metals occurs below the ductile-to-brittle transition temperature (DBTT), and is promoted by constrained stress-states, higher strain rates and lower temperatures, all of which act to inhibit plastic flow. High symmetry systems, such as face-centered cubic metals, however, do not generally cleave and only fail by a microscopically brittle fracture mode when the grain 
boundaries are embrittled, e.g., due to the segregation of impurities there or from the presence of trapped hydrogen.

For brittle fracture, lower strain hardening is generally preferential for toughness as it limits the elevation of stresses at a crack tip; microstructurally finer grain sizes and smaller second-phase particles (e.g., inclusions), coupled with the absence of segregated species at grain boundaries or other internal interfaces, all tend to promote higher intrinsic toughness For ductile fracture, conversely, high strain hardening is preferred as it provides a steady source of hardening to suppress strain localization (e.g., necking) ${ }^{[*]}$ which in turn creates a wider distribution of damage; microstructurally, distributions of finer second-phase particles again provide greater resistance to fracture.

Ceramic materials: In contrast to metallic materials, at low homologous temperatures most structural ceramics, such as $\mathrm{Al}_{2} \mathrm{O}_{3}, \mathrm{ZrO}_{2}, \mathrm{SiC}$ and $\mathrm{Si}_{3} \mathrm{~N}_{4}$, suffer from almost a complete lack of plastic deformation; this is due to the absence of mobile dislocation activity, although other modes of inelastic deformation, such as microcracking and in situ phase transformation, can provide limited alternative deformation mechanisms. The implications from this are that ceramics are inherently brittle with an extreme sensitivity to flaws. Actually, they are essentially impossible to toughen intrinsically; in the absence of extrinsic shielding mechanisms, fracture invariably occurs catastrophically (with crack initiation concomitant with instability) by cohesive bond breaking at the crack tip with a resulting very low (intrinsic) toughness of roughly 1 to $3 \mathrm{MPa} \sqrt{\mathrm{m}}$.

Toughening ceramics, as with virtually all brittle materials, must be achieved extrinsically, i.e., through the use of microstructures which can promote crack-tip shielding mechanisms such as crack deflection, in situ phase transformations, constrained microcracking (although this mechanism is generally not too potent), and most importantly crack bridging. As described above, extrinsic mechanisms result in R-curve behavior as they operate primarily behind the crack tip to lessen the effective crack-driving force; they are therefore mechanisms of crackgrowth toughening. Since the critical stress intensity $K_{\mathrm{c}}$ is proportional to the $\sigma_{\mathrm{F}} V_{a}$,

${ }^{[*}$ This is the basis of toughening by "transformation-induced plasticity", as for example seen in certain ductile steels that display an in situ martensitic phase transformation (TRIP steels); the in situ transformation from the softer austenite to the harder martensite phase provides a steady source of hardening with increasing deformation which acts to delay strain localization. The effect is quite distinct from "transformation toughening" that is found in certain brittle materials, such as zirconia-based ceramics, that also undergo in situ phase transformations (as described below). 
the fracture stress, $\sigma_{\mathrm{F}}$, can be deduced from the R-curve with a knowledge of the prevalent flaw sizes, $a$. As discussed in detail elsewhere, ${ }^{[8]}$ in addition to the peak toughness, it is the early proportion of the R-curve (over the first hundred micrometers or so of crack extension) that is also very important for ceramics as this governs their fracture strength at realistic flaw sizes. Consequently, the resulting strengths depend markedly on the details of the R-curve and of course the initial flaw sizes, such that optimizing strength vs. toughness can once again involve different choices of microstructures.

Extrinsic toughening mechanisms relevant to many monolithic and composite ceramics and the more brittle intermetallics can be categorized into several distinct classes ${ }^{[6,7]}$ namely, crack-tip shielding from (i) crack deflection and meandering, (ii) inelastic or dilated zones surrounding the wake of the crack, (zone shielding), (iii) wedging, bridging and/or sliding between crack surfaces, (contact shielding), and (iv) combinations thereof. Extensive reviews of these mechanisms can be found elsewhere; ${ }^{[6,7]}$ in essence they toughen by lowering the local or near-tip stress intensity, $K_{\text {tip }}$, actually experienced at the crack tip, relative to the globally applied value, $K_{\mathrm{app}}$, i.e., $K_{\text {tip }}=K_{\mathrm{app}}-K_{\mathrm{s}}$, where $K_{\mathrm{s}}$ is the stress intensity generated by the shielding mechanism. For example in transformationtoughened ceramics, such as partially-stabilized zirconia, ${ }^{[9,10]}$ the 2 to $4 \%$ dilation associated with an in situ martensitic transformation from the tetragonal to monoclinic $\mathrm{ZrO}_{2}$ phase generates a crack-tip transformed zone that is in compression (due to the surrounding constraint of untransformed material); as the crack extends into this zone, the near-tip stress intensity is progressively reduced leading to a rising $\mathrm{R}$-curve where the peak (steady-state) toughness may approach $15 \mathrm{MPa} \sqrt{\mathrm{m}}$, a factor of some five times larger than the intrinsic toughness. A more omnipresent example of shielding is crack bridging, which is found in different forms in almost all classes of materials, particularly natural and biological materials. Here intact "features" span the crack as it opens thereby carrying load that would be otherwise used to extend the crack. These "features" include certain reinforcements, e.g., fibers or ductile phases in composites, "uncracked ligaments" between a main crack and (micro)cracks initiated ahead of it, which a common toughening mechanism in ceramics, rocks and biological materials like bone and tooth dentin, and frictional bridging associated with interlocking grains during intergranular fracture, which is the primary and most potent source of toughening in structural ceramics with engineered grain boundaries, e.g., $\mathrm{Al}_{2} \mathrm{O}_{3}, \mathrm{Si}_{3} \mathrm{~N}_{4}$, $\mathrm{SiC}$. ${ }^{[11-18]}$

Polymeric materials: Polymers do not contain crystallographic planes, dislocations, and grain boundaries but rather consist of (generally) covalently-bonded molecular networks, which in thermoplastic polymers are in the form of long 
chains held loosely together by secondary van der Walls bonds. Ultimate fracture normally requires breaking the primary bonds, but the secondary bonds often play a major role in the deformation mechanisms that lead to fracture. As they are generally rate-dependent materials, factors such as strain rate, temperature and molecular structure have a strong influence on ductility and toughness in polymers. At high rates/low temperatures (relative to the glass transition temperature), polymers tend to be brittle, as there is insufficient time for yielding or larger-scale viscoelastic mechanisms to respond to stress. Shear yielding and crazing are competing mechanisms here. Shear yielding resembles plastic flow in metals; molecules slide with respect to one another when subjected to critical shear stress. Crazing, which occurs in glassy polymers subjected to tensile stresses, represents highly localized deformation that leads to cavitation (void formation); compared to shear yielding, crazes are more likely ahead of a crack tip because of the triaxial stress-state there. The crack advances when the fibrils at the trailing edge of the craze rupture. In other words, cavities in the craze zone coalesce with the crack tip, similar to microvoid coalescence in metals. Craze crack growth can either be stable or unstable, depending on the relative toughness of the material.

Composite materials: The incorporation of reinforcements in the form of fibers, whiskers or particles can also toughen materials, although the motivation may be rather to increase strength and/or stiffness. For toughening, crack bridging is again the most prominent mechanism, particularly in ceramic-matrix composites; by utilizing fibers with weak fiber/matrix bonding, when the matrix fails, the fibers are left intact spanning the crack wake and can act as bridges to inhibit crack opening. ${ }^{[7]}$ Analogous toughening in metal-matrix composites is considerably less advanced, in part because many such composites are designed with strong reinforcement-matrix interfaces and thus do not develop crack bridging to any significant degree. In metal-matrix composites discontinuously reinforced with a brittle particulate phase, such as SiC-aluminum alloy composites, the intent is to increase the strength and sometimes wear resistance, yet toughening by crack bridging can result from the uncracked ligaments created where microcracks, formed at $\mathrm{SiC}$ particles some distance ahead of the crack tip, have yet to link to the main crack. In continuous-fiber reinforced polymer composites which are expensive yet seeing ever increasing use in aerospace structures and in other lightweight structural applications, high volume fractions of graphite fibers, with strong matrix/fiber interfaces, are added for strength and stiffness; to a lesser degree, continuous glass and ceramic fibers have also been used for improved strength. For discontinuous reinforcements, additions of rubber particles can promote both crack deflection and bridging, as 
the crack will tend to follow the low modulus rubber phase, which while it remains intact can act as a particle bridge across the crack; this is the basis of rubber-toughened polymers.

A far more controversial notion in composite toughening is the use of nanoscale reinforcements such as carbon nanotubes (with their near-ideal strength and extreme stiffness) as toughening reinforcements. It has been claimed that composites made with such nanoscale reinforcing materials as nanotubes, platelets and nanofibers would have exceptional properties; however, results to date have been disappointing. ${ }^{[19]}$ If the composite material is to be used for a small-volume structure, clearly the reinforcements must also be small; moreover, as there is a lessened probability of finding defects, small-volume reinforcements tend to be much stronger, as has been known since the early days of research on whiskers. ${ }^{[20]}$ However, from the perspective of toughening, we would strongly argue that nanoscale reinforcements are not the best direction to take. The prime extrinsic toughening mechanisms, namely crack deflection and particularly crack bridging, are promoted by increasing, not decreasing, reinforcement dimensions. ${ }^{[7]}$

\subsection{Toughening in biological materials}

Although structure defines mechanical properties, specific properties are controlled by nano/microstructure at widely differing length-scales. Nowhere is this more apparent than with biological materials, which are invariably sophisticated composites whose unique combination of mechanical properties derives from architectural design that spans multiple dimensions. Biological organisms produce composites that are organized in terms of composition and structure, containing both inorganic and organic components in complex anisotropic arrangements. Using materials available in their environment that typically exhibit poor macroscale mechanical properties (brittle biological ceramics and compliant macromolecules), Nature can create composite structures that are hierarchically organized at the nano, micro and meso levels to achieve orders of magnitude increases in strength and toughness compared to their constituent phases. ${ }^{[21]}$ Some hard mineralized biocomposites including bone, dentin and nacre further exhibit high degree of inelasticity, despite the brittle nature of their constituents.

From a fracture mechanics perspective, it is clear that most of these biological materials derive their fracture resistance through the presence of a series of extrinsic toughening mechanisms acting at various length-scales; as with 
synthetic materials, this results in characteristic R-curve behavior with the vital implication that these materials develop most of their toughening during crack growth, not during crack initiation. We examine now the specific behavior of three such natural materials, namely nacre, bone and wood.

Nacre: Nacre (abalone shell) consists of a fine-scale layered brick-like structure comprising $\sim 95$ vol.\% of submicron $(\sim 500 \mathrm{~nm})$ layered aragonite $\left(\mathrm{CaCO}_{3}\right)$ platelets bonded by a thin $(20-30 \mathrm{~nm})$ layer of organic protein material. Despite the low toughness of either of its constituents, nacre displays a fracture toughness (in energy terms) that is roughly an order of magnitude higher than either the aragonite or the proteinous layer. ${ }^{[22-24]}$ This is achieved, however, not simply through multi-dimensional architectural design but also using precisely and carefully design interfaces. Specifically, the hard aragonite platelets provide for strength but are brittle; inter-lamellae shear in the proteinous layers provides sufficient inelastic deformation to permit redistribution of stress around strainconcentration sites. ${ }^{[25]}$ In fact, the prominent multi-scale toughening mechanisms in nacre occur through viscoplastic energy dissipation within this biopolymer layer where the tablets are able to engage in limited slip over one another. Suggested models include $(i)$ organic layer acting as a viscoelastic glue involving breakage of sacrificial bonds in the biopolymer at the molecular level[26], (ii) inelastic shearing resisted by the interface roughness nano-asperities ${ }^{[25]}$, (iii) "plastic deformation" at the nanometer level inside individual aragonite platelets $^{[27]}$ (although we personally find this hard to believe), and (iv) at the micrometer level, the breaking of aragonite mineral bridges which exist between the mineral layers. ${ }^{[21,28]}$

Bone: Bone is a hierarchical composite of a fibrous polymer (collagen) and hard mineral nanoparticles (carbonated hydroxyapatite) that that is imbued with mechanisms to resist fracture at multiple size-scales. ${ }^{[29]}$ These size-scales relate to the characteristic structural dimensions in bone, which vary from twisted peptide chains at the nanoscale, hydroxyapatite-impregnated twisted collagen fibrils at the scale of tens of nanometers, collagen fibers that are typically a micrometer in diameter, the lamellar structure of these fibers above micrometer dimensions, to the (secondary) osteon (Haversian) structures, which are several hundred micrometers in size. It is the simultaneous operation of toughening mechanisms at these various length scales that provides bone with its enduring strength and toughness. Several microscale toughening mechanisms have been reported for cortical bone, including sacrificial bonding between fibrils, viscoplastic flow, ${ }^{[30]}$ constrained microcracking, ${ }^{[31]}$ crack deflection ${ }^{[32]}$ and crack bridging, ${ }^{[33]}$ although the most critical of these for the macroscopic fracture 
toughness appear to be crack deflection and bridging. At their most potent, both these latter mechanisms operate in human cortical bone at dimensions between several to several hundred micrometers and are motivated by preferred microcracking along the interfaces of the osteons ("cement lines"). As the cement lines are orientated along the axis of the bone, bone is much easier to split than to break. In the longitudinal (splitting) direction, cement line microcracks form parallel to the direction of fracture; the uncracked regions in between provide for toughening by uncracked-ligament bridging. In the transverse (breaking) direction, conversely, the primary microcracks are orientated nominally perpendicular to the main crack path; crack deflection at these weak interfaces now results in local crack arrest and interface delamination (the Cook-Gordon mechanism ${ }^{[34]}$ ) resulting in much higher (R-curve) toughening. Indeed, based on recent measurements on physiologically relevant short (sub-millimeter) cracks, the stress intensity needed to fracture human bone is more than five times higher in the transverse direction than in the longitudinal direction. ${ }^{[19]}$

Wood: Wood has high specific stiffness (stiffness per unit weight) and specific strength that is comparable with steel. ${ }^{[35]}$ The outstanding mechanical properties are mainly due to the hierarchical structure and optimized reinforcement orientation of cellulose fibrils. Wood is a cellular composite with four levels of hierarchical structure: molecular, fibrillar, cellular and macroscopic structure. The main structural constituent of wood is cellulose, a high molecular weight polysaccharide which contributes to its stiffness and strength. The cellulose is organized into microfibrils, $\sim 10-20 \mathrm{~nm}$ in diameter, that comprise both crystalline and amorphous regions. Bundles of cellulose microfibrils further form macrofibrils which are embedded in an amorphous matrix of lignin, hemicellulose and other compounds. A most remarkable property of wood is highly anisotropic fracture toughness; moreover, its highest toughness is ten times larger than that of a fibrous composite with the same fraction of fibers and matrix. Fiber pull-out is mainly responsible for this high toughness. ${ }^{[36]}$ Shear cracks open and propagate longitudinally, which allows each cell wall to be pulled apart without being broken through. Whereas it is easy for a crack to propagate parallel to the grain, perpendicular propagation is very difficult as cracking is impeded by the weak interfaces. Such anisotropic toughening is analogous to that in bone and has similarities to that in nacre when the shell is loaded along the direction of its surface; the individual platelets are "pulled out" which again provides a prime source of toughening. ${ }^{[21]}$ 


\section{Designing high toughness bioinspired structural materials}

It is apparent from the forgoing descriptions that compared to traditional engineering, Nature develops strong and tough materials in far more complex fashion, using hierarchical design and principles of biologically controlled selfassembly; in addition, many such natural materials can remodel and adapt in service to changing environmental conditions. Synthetic structural materials that could mimic such natural design could transform many engineering disciplines, especially the energy-related and transportation industries. ${ }^{[37]}$ Indeed, it has been acknowledged that control over structure at all hierarchal levels is the key to the successful use of polymers and composites as structural materials. ${ }^{[29]}$ Accordingly, the notion of replicating natural designs in engineering composites has generated enormous interest. ${ }^{[38-41]}$ However, in reality the biomimetic approach has yielded few technological advances, in large part because of the lack of processing techniques able to achieve such complex structural hierarchy in practical dimensions. For example, fabricating a layered nacre-like composite with sub-micrometer mineral layers can be achieved by physical or chemical deposition, but not as bulk materials as these techniques are restricted to the fabrication of thin films. Indeed, the design and actual fabrication of synthetic biologically-inspired bulk materials remains one of the "grand challenges" in materials science.

Some progress has been made along these lines recently with the development of a range of bone- and nacre-like bulk structural materials with high inorganic (ceramic) content, consisting of complex lamellar and/or "brick-and-mortar" like structures as fine-scale stiff/hard ceramic lamellae separated by thin (submicronscale), soft, lubricating polymeric layers. ${ }^{[42]}$ Fabricated using an ice-templating process, ${ }^{[42]}$ these hybrid materials are designed to induce multiple toughening mechanisms operating over sub-micrometer to millimeter-scale dimensions; one intent is to precisely control the extent of inter-lamellae shear by careful design of the surface roughness of the layers and the ceramic "bridges" that may connect them. Using just simple constituents, e.g., alumina and PMMA, the mechanical behavior of these synthetic composites can actually be made to replicate natural materials; in terms of properties, at a strength level of $200 \mathrm{MPa}$, extensive Rcurve toughening has been obtained in $\sim 80 \%$ ceramic structures with measured $K_{\mathrm{c}}$ toughnesses in excess of $30 \mathrm{MPa} \sqrt{ }$, i.e., representing more than an order of magnitude increase in the toughness as compared to either of the constituent phases (Fig. 3). ${ }^{[42]}$ 


\section{Closure}

Modern structural materials are increasingly tailored to have optimum combinations of properties for specific applications. The fracture toughness is invariably a critical material parameter for many such structural applications; indeed, few advanced materials are "strength-challenged", it is the fracture properties that are invariably limiting. In this overview, we have briefly described how the fracture resistance of many engineering and biological materials is intimately related to their microstructure through two main classes of toughening (intrinsic and extrinsic) mechanisms. Many such materials are toughened extrinsically, by crack-tip shielding mechanisms which result in rising R-curve behavior, i.e., they develop their primary toughening during crack growth, rather than for crack initiation. While single-value parameters based on

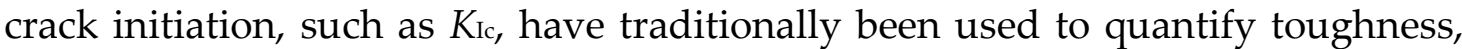
they cannot capture, nor even adequately represent, the toughening in these classes of materials, where a full R-curve determined is warranted. Examples are given from both engineering and biological materials, the latter providing the best demonstration of this with their ability to derive multiple extrinsic toughening mechanisms over a wide range of length-scales. It remains to be seen how successful we can be in mimicking Nature's design in the development of both strong and tough synthetic materials with this multi-dimensional hierarchical approach.

\section{References}

[1] J. Liu, A. G. Rinzler, H. J. Dai, J. H. Hafner, R. K. Bradley, P. J. Boul, A. Lu, T. Iverson, K. Shelimov, C. B. Huffman, F. Rodriguez-Macias, Y. S. Shon, T. R. Lee, D. T. Colbert, R. E. Smalley, Science 1998, 280, 1253.

[2] K. T. V. Rao, R. O. Ritchie, Int. Mater. Rev. 1992, 37, 153.

[3] Y. Kimura, T. Inoue, F. X. Yin, K. Tsuzaki, Science 2008, 320, 1057.

[4] W. B. Parsons, Engineers and Engineering in the Renaissance, Williams \& Wilkins Co., Baltimore, MD 1939.

[5] J. F. Knott, Fundamentals of fracture mechanics, Butterworth \& Co. (Publishers) Ltd, London, U.K. 1976.

[6] R. O. Ritchie, Mater. Sci. Eng. A 1988, 103, 15.

[7] A. G. Evans, J. Am. Ceram. Soc. 1990, 73, 187.

[8] J. J. Kruzic, R. L. Satet, M. J. Hoffmann, R. M. Cannon, R. O. Ritchie, J. Am. Ceram. Soc. 2008, 91, 1986. 
[9] R. H. Dauskardt, W. Yu, R. O. Ritchie, J. Am. Ceram. Soc. 1987, 70, C248.

[10] R. H. Dauskardt, D. B. Marshall, R. O. Ritchie, J. Am. Ceram. Soc. 1990, 73, 893.

[11] F. F. Lange, J. Am. Ceram. Soc. 1973, 56, 518.

[12] P. L. Swanson, C. J. Fairbanks, B. R. Lawn, Y. W. Mai, B. J. Hockey, J. Am. Ceram. Soc. 1987, 70, 279.

[13] Y. W. Mai, B. R. Lawn, J. Am. Ceram. Soc. 1987, 70, 289.

[14] N. P. Padture, B. R. Lawn, J. Am. Ceram. Soc. 1994, 77, 2518.

[15] J. J. Cao, W. J. MoberlyChan, L. C. DeJonghe, C. J. Gilbert, R. O. Ritchie, J. Am. Ceram. Soc. 1996, 79, 461.

[16] P. F. Becher, E. Y. Sun, K. P. Plucknett, K. B. Alexander, C. H. Hsueh, H. T. Lin, S. B. Waters, C. G. Westmoreland, E. S. Kang, K. Hirao, M. E. Brito, J. Am. Ceram. Soc. 1998, 81, 2821.

[17] J. J. Kruzic, R. M. Cannon, J. W. Ager, R. O. Ritchie, Acta Mater. 2005, 53, 2595.

[18] J. J. Kruzic, R. M. Cannon, R. O. Ritchie, J. Am. Ceram. Soc. 2004, 87, 93.

[19] K. J. Koester, J. W. Ager, R. O. Ritchie, Nat. Mater. 2008, 7, 672.

[20] A. Kelly, Strong Solids, Clarendon Press, Oxford, UK 1966.

[21] M. A. Meyers, P. Y. Chen, A. Y. M. Lin, Y. Seki, Prog. Mater Sci. 2008, 53, 1.

[22] J. D. Currey, Proc. R. Soc. London, Ser. B 1977, 196, 443.

[23] A. P. Jackson, J. F. V. Vincent, R. M. Turner, Proc. R. Soc. London, Ser. B 1988, 234, 415.

[24] F. Barthelat, H. D. Espinosa, Exp. Mech. 2007, 47, 311.

[25] A. G. Evans, Z. Suo, R. Z. Wang, I. A. Aksay, M. Y. He, J. W. Hutchinson, J. Mater. Res. 2001, 16, 2475.

[26] B. L. Smith, T. E. Schaffer, M. Viani, J. B. Thompson, N. A. Frederick, J. Kindt, A. Belcher, G. D. Stucky, D. E. Morse, P. K. Hansma, Nature 1999, 399, 761.

[27] X. D. Li, W. C. Chang, Y. J. Chao, R. Z. Wang, M. Chang, Nano Lett. 2004, 4, 613.

[28] F. Song, A. K. Soh, Y. L. Bai, Biomaterials 2003, 24, 3623.

[29] P. Fratzl, R. Weinkamer, Prog. Mater Sci. 2007, 52, 1263.

[30] G. E. Fantner, T. Hassenkam, J. H. Kindt, J. C. Weaver, H. Birkedal, L. Pechenik, J. A. Cutroni, G. A. G. Cidade, G. D. Stucky, D. E. Morse, P. K. Hansma, Nat. Mater. 2005, 4, 612 .

[31] D. Vashishth, K. E. Tanner, W. Bonfield, J. Biomech. 2003, 36, 121.

[32] H. Peterlik, P. Roschger, K. Klaushofer, P. Fratzl, Nat. Mater. 2006, 5, 52. 
[33] R. K. Nalla, J. J. Kruzic, J. H. Kinney, R. O. Ritchie, Biomaterials 2005, 26, 217.

[34] J. Cook, C. C. Evans, J. E. Gordon, D. M. Marsh, Proceedings of the Royal Society of London Series a-Mathematical and Physical Sciences 1964, 282, 508.

[35] U. G. K. Wegst, M. F. Ashby, Philos. Mag. 2004, 84, 2167.

[36] G. Jeronimidis, Proc. R. Soc. London, Ser. B 1980, $208,447$.

[37] C. Ortiz, M. C. Boyce, Science 2008, 319, 1053.

[38] A. Sellinger, P. M. Weiss, A. Nguyen, Y. F. Lu, R. A. Assink, W. L. Gong, C. J. Brinker, Nature 1998, 394, 256.

[39] Z. Y. Tang, N. A. Kotov, S. Magonov, B. Ozturk, Nat. Mater. 2003, 2, 413.

[40] P. Podsiadlo, A. K. Kaushik, E. M. Arruda, A. M. Waas, B. S. Shim, J. D. Xu, H. Nandivada, B. G. Pumplin, J. Lahann, A. Ramamoorthy, N. A. Kotov, Science 2007, 318,80 .

[41] L. J. Bonderer, A. R. Studart, L. J. Gauckler, Science 2008, 319, 1069.

[42] E. Munch, M. E. Launey, D. H. Alsem, E. Saiz, A. P. Tomsia, R. O. Ritchie, Science 2008, In Press. 


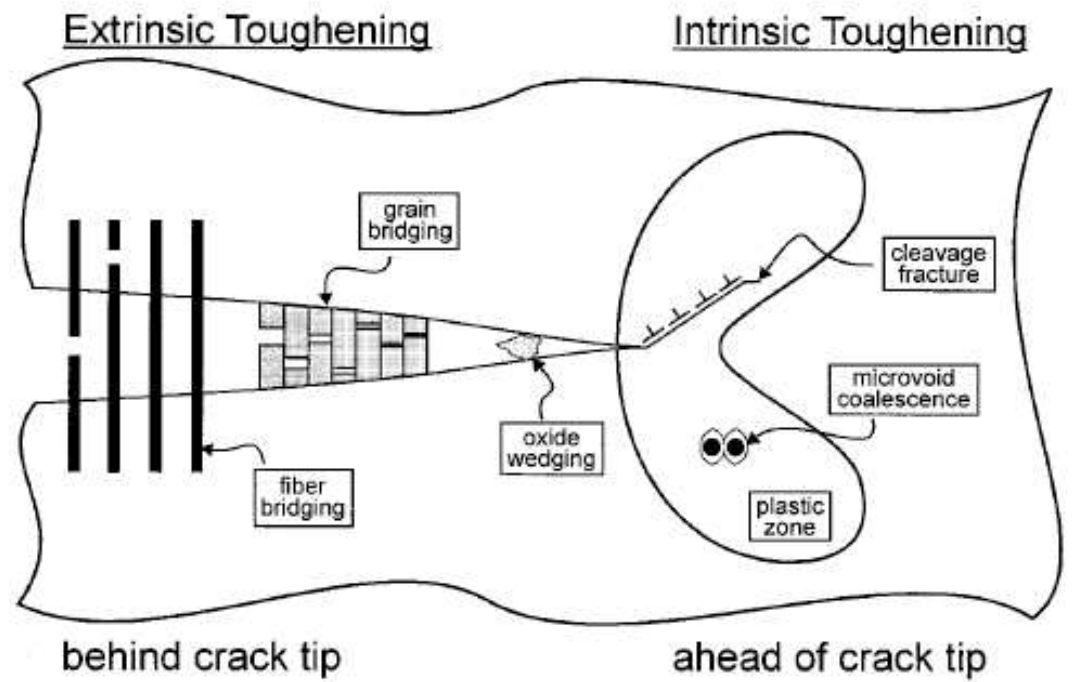

Figure 1: The notion of extrinsic vs. intrinsic toughness. Schematic illustration of the mutual competition between intrinsic mechanisms of damage which act ahead of the crack tip to promote crack advance and extrinsic mechanisms of crack-tip shielding that act mainly behind the crack tip to impede crack advance. Extrinsic toughening mechanisms result in crack-resistance curve (R-curve) behavior and affect only the crack-growth toughness. ${ }^{[6]}$ 

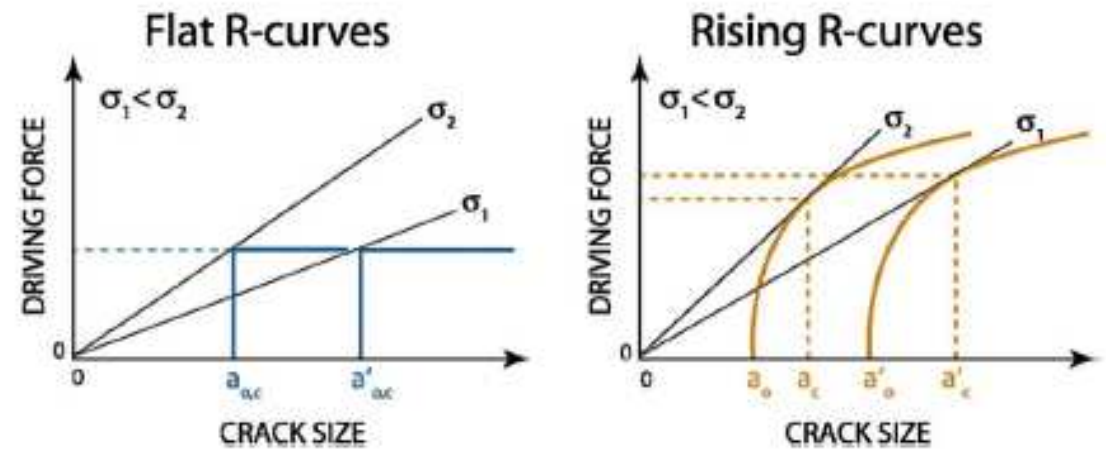

Figure 2: Crack-resistance curves. Schematic illustrations of flat and rising crackgrowth resistance curves (R-curves). Unstable fracture occurs when the materials resistance to fracture ceases to increase faster than the driving force for crack propagation; this corresponds to the driving force as a function of crack size being tangent to the crack-growth resistance curve. For a material that has a flat R-curve, a single value of toughness unambiguously characterizes the material. For a material with a rising R-curve there is no single value of toughness that characterizes the material as the driving force for unstable crack propagation depends on the extent of crack growth. For materials with rising R-curves, measurements are needed to determine how the resistance to fracture evolves with crack extension. For materials with flat R-curves, there is no stable crack extension and the initial crack size $\left(a_{0}\right)$ is the same as the critical crack size $\left(a_{c}\right)$. In materials with a rising R-curve, stable crack growth occurs and the critical crack size will be larger than the initial crack size. Crack-growth resistance curves are typically plotted with crack extension $(\Delta a)$ instead of crack size because the shape of the R-curve does not vary with crack size. The driving force for crack propagation can be quantified by such characterizing parameters as $K, \mathcal{G}$, or $J$. 

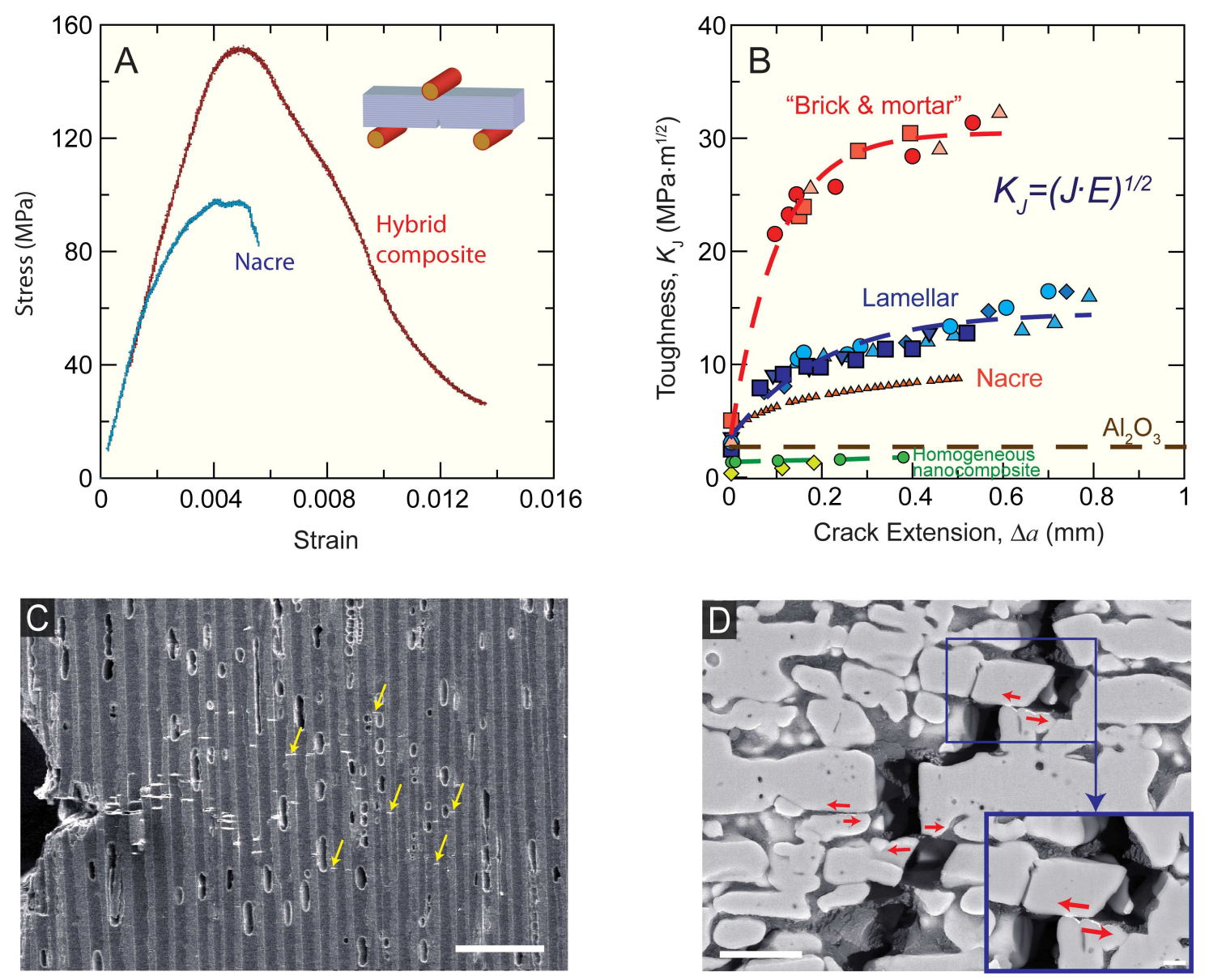

Figure 3: Mechanical response and toughening mechanisms in the biomimetic composites. (A) Three-point bending stress-strain curves for $\mathrm{Al}_{2} \mathrm{O}_{3} / \mathrm{PMMA} \mathrm{Pybrid}$ materials can be seen to mimic those of nacre, and show extensive inelastic deformation prior to failure. The curves correspond to lamellar hybrid composite and hydrated nacre from abalone shell. (B) These biomimetic materials show exceptional crack growth toughness, akin to that observed in natural composites, and hence display a significant increase in the crack-resistance with crack extension (R-curve behavior). By comparison, negligible toughening is observed in nanocomposites consisting of $500 \mathrm{~nm} \mathrm{Al} \mathrm{Al}_{2} \mathrm{O}_{3}$ particles dispersed in PMMA. (C) Scanning electron micrograph taken during an in situ R-curve measurement of a lamellar structure shows two of the toughening mechanisms acting at large scales: the wide distribution of damage (over millimeter dimensions) in the form of contained microcracking within the ceramic layers (yellow arrows point to some of these microcracks) and the voids in the polymer layers. (D) In situ imaging of crack propagation in "brick-and-mortar" structures shows clear evidence of "pull out" and frictional sliding between ceramic bricks (see also insert). The thin bright lines between the sliding grains in the inset indicate electrical charging in the SEM resulting from the deformation of the gold coating during sliding. The bars are $250 \mu \mathrm{m}$ in (C), 10 $\mu \mathrm{m}$ in (D), and $2 \mu \mathrm{m}$ in the insert in (B). ${ }^{[42]}$ 